\title{
Modeling of phytochrome absorption spectra
}

\author{
Olle Falklöf and Bo Durbeej
}

\section{Linköping University Post Print}

\section{Tweet}

N.B.: When citing this work, cite the original article.

This is the pre-reviewed version of the following article:

Olle Falklöf and Bo Durbeej, Modeling of phytochrome absorption spectra, 2013, Journal of Computational Chemistry, (34), 16, 1363-1374.

which has been published in final form at:

http://dx.doi.org/10.1002/jcc.23265

Copyright: Wiley-Blackwell

http://eu.wiley.com/WileyCDA/Brand/id-35.html 


\title{
Modeling of Phytochrome Absorption Spectra
}

\author{
Olle Falklöf and Bo Durbeej* \\ Division of Computational Physics, IFM, Linköping University, SE-581 83 Linköping, Sweden \\ E-mail: bodur@ifm.liu.se
}




\begin{abstract}
Phytochromes constitute one of the six well-characterized families of photosensory proteins in Nature. From the viewpoint of computational modeling, however, phytochromes have been the subject of much fewer studies than most other families of photosensory proteins, which is likely a consequence of relevant high-resolution structural data becoming available only in recent years. In this work, hybrid quantum mechanics/molecular mechanics (QM/MM) methods are used to calculate UV-vis absorption spectra of Deinococcus radiodurans bacteriophytochrome. We investigate how the choice of QM/MM methodology affects the resulting spectra and demonstrate that QM/MM methods can reproduce the experimental absorption maxima of both the Q and Soret bands with an accuracy of about $0.15 \mathrm{eV}$. Furthermore, we assess how the protein environment influences the intrinsic absorption of the bilin chromophore, with particular focus on the Q band underlying the primary photochemistry of phytochromes.
\end{abstract}

Keywords: Photosensory proteins, Bilin chromophores, QM/MM methods, Timedependent density functional theory 


\section{Graphical Table of Contents}

Hybrid quantum mechanics/molecular mechanics (QM/MM) methods are used to calculate UV-vis absorption spectra of a photosensory protein belonging to the phytochrome family. Besides providing valuable information on how the choice of QM/MM methodology affects the resulting spectra, the article also sheds new light on how the protein tunes the absorption of its bilin chromophore.

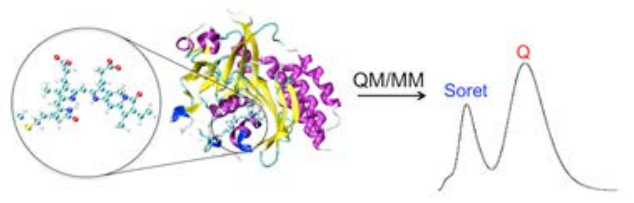




\section{Introduction}

Phytochromes are photosensory proteins that based on light conditions regulate physiological and developmental processes in plants, bacteria, cyanobacteria and fungi. ${ }^{[1-11]}$ Several structural motifs are shared between the members of the phytochrome family, such as a linear tetrapyrrole (bilin) chromophore that absorbs light in the red/farred region and is covalently attached to a cysteine residue of the apoprotein through a thioether linkage. ${ }^{[3]}$ Apart from the characteristic strong absorption in the red/far-red region (the so-called Q band), the UV-vis spectra of phytochromes also contain a peak in the blue region (the so-called Soret band). Through the absorption of red/far-red light, an equilibrium between the two distinct photochromic Pr (red-absorbing) and Pfr (far-redabsorbing) forms of the protein is attained, where the relative concentrations of the two forms govern a variety of processes in the host organism. ${ }^{[1]}$ In most phytochromes, the dark-adapted inactive state is dominated by the Pr form, whereas the physiologically active state contains higher concentrations of the Pfr form. A few examples of processes regulated by phytochromes are seed germination, flowering time and shade avoidance in plants, ${ }^{[7]}$ and phototaxis in bacteria. ${ }^{[10]}$

Despite much progress in recent years, understanding the molecular mechanism of the $\mathrm{Pr} \rightarrow$ Pfr conversion, which proceeds via several metastable intermediates ${ }^{[12]}$ and is triggered by an excited-state isomerization of the bilin chromophore, ${ }^{[1,13-17]}$ remains a very active field of research. For example, this problem has been investigated by mutational studies, ${ }^{[18-21]}$ X-ray crystallography ${ }^{[21-28]}$ and various forms of spectroscopy, ${ }^{[15-17,29-39]}$ such as resonance Raman $(\mathrm{RR})^{[15,16,29,30,32,33]}$ and NMR. ${ }^{[37-39]}$ Furthermore, a number of contributions have also been made by computational studies investigating, e.g., the intrinsic photochemical reactivity ${ }^{[40-44]}$ and protonation state ${ }^{[45,46]}$ of the bilin chromophore, as well as the relationship between light absorption and chromophore conformation. ${ }^{[47-50]}$ These studies employed quantum chemical methods and typically focused on chromophore properties in either the gas phase or in solution, representing the solvent using an implicit reaction-field model. Thus, the impact of specific interactions between the chromophore and the surrounding protein was not accounted for. 
In order to afford quantum chemical modeling of electronic processes in large biological systems, one common approach is to use a hybrid quantum mechanics/molecular mechanics (QM/MM) method. ${ }^{[51-53]}$ Thereby, the quantum chemical description of the chemically reactive parts of the system is coupled to a less expensive, classical force-field description of the environment. While this approach has been used to model excitation energy transfer in bilin-chromophore-containing antenna proteins involved in photosynthesis ${ }^{[54-56]}$ and to calculate RR spectra of phytochromes, ${ }^{[57,58]}$ its application to UV-vis spectra of phytochromes has not been investigated in greater detail, although a preliminary study focusing on the lowest excited state of a bacteriophytochrome has been reported. ${ }^{[42]}$ By using QM/MM methods to systematically compute the Q- and Soret-band absorption maxima of Deinococcus radiodurans bacteriophytochrome (DrBphP) (Figure 1), ${ }^{[20,23]}$ which harbors a biliverdin IX $\alpha$ (BV) chromophore (Figure 2) and for which crystallographic data are available, ${ }^{[22,23]}$ the present study is aimed at filling this gap. As such, the study is related to previous investigations concerned with QM/MM-based modeling of UV-vis spectra of other photosensory proteins like the rhodopsins, ${ }^{[59-63]}$ photoactive yellow protein ${ }^{[64,65]}$ and the flavin-containing BLUF and phototropin proteins. ${ }^{[66-68]}$ Besides providing valuable information on how the choice of QM/MM methodology affects the accuracy of the computed absorption maxima, our study will also help illuminate how the protein environment influences the light absorption of the BV chromophore. Taken together, these results will facilitate future QM/MM-based studies of key photochemical problems in phytochrome research, such as establishing the chromophore isomerization mechanism. ${ }^{[15-17]}$

Although the bilin chromophores of plant, bacterial, cyanobacterial and fungal phytochromes have different conjugation lengths, they are chemically similar, which suggests that this work will have bearing beyond the specific phytochrome ( $\mathrm{DrBphP})$ studied. At the same time, however, distinct differences between different phytochrome family members have indeed been reported. For example, based on circular dichroism spectroscopy and quantum chemical calculations, it has been shown that the Pfr states of DrBphP and the cyanobacterial phytochrome Cph1 are structurally dissimilar, and inferred that the directionality of chromophore isomerization is different in these two 
phytochromes. ${ }^{[69]}$ Furthermore, although it is widely accepted that the light-induced chromophore isomerization involves rotation of the $\mathrm{D}$ pyrrole ring (Figure 2), ${ }^{[13-15,28,30,32,33]}$ which for the case of a bacteriophytochrome has been clearly demonstrated through cryocrystallographic characterization of reaction intermediates, ${ }^{[28]}$ NMR data on the solution structure of $\mathrm{Cph} 1$ is more consistent with rotation of the A ring. ${ }^{[39]}$ Overall, then, a natural goal of future computational studies in this field will also be to investigate how QM/MM methods apply to a range of different phytochromes, as well as to related biliprotein photosensors like the shorter-wavelength-absorbing cyanobacteriochromes found in cyanobacteria. ${ }^{[0,71]}$

\section{Computational Details}

\section{Preparation of the protein model}

Atomic coordinates were taken from the high-resolution (1.45 $\AA$ ) crystal structure of the chromophore binding domain of $\mathrm{DrBphP}$ in the Pr form (Protein Data Bank [PDB] entry: $2 \mathrm{O} 9 \mathrm{C}){ }^{[23]}$ which is an improvement of the first-ever crystal structure (solved at $2.50 \AA$ resolution) ${ }^{[22]}$ reported of a phytochrome. In addition to the BV chromophore, which adopts a C5-Z,syn C10-Z,syn C15-Z,anti (ZsZsZa) conformation, the structure contains 319 amino acids (residues 4 to 322 in the polypeptide chain) and several water molecules. In the protein-structure refinement process, some of the atoms were assigned partial occupancies at two different sites, designated A and B. In general, it is difficult to assess which particular combination of designations is most relevant for the corresponding atoms based on standard refinement techniques alone. However, since most of these atoms reside at such distances ( $>8 \AA$ ) away from the chromophore that their influence on the light absorption is expected to be minor, the A coordinates were invoked without further consideration. To account for the fact that residues Asp4, Arg70, Glu193, His196 and $\operatorname{Arg} 310$ are truncated after the $\beta$ carbon in the crystal structure, these residues were treated as alanines.

The structure deposited in the PDB does not contain hydrogen atoms. ${ }^{[23]}$ Instead, protonation states of titratable residues were determined based on $\mathrm{p} K_{\mathrm{a}}$ values estimated 
using PROPKA 2.0. ${ }^{[72,73]}$ Thereby, all arginine and lysine residues were found to be protonated, and all aspartate and glutamate residues were found to be deprotonated. Moreover, the estimated $\mathrm{p} K_{\mathrm{a}}$ values indicated that only one (His322) out of the 12 histidines is protonated. The question as to which tautomeric form (hydrogen on the $\delta 1$ or $\varepsilon 2$ nitrogen) is most relevant for the 11 neutral histidines was approached by following the arguments of Wagner et al., ${ }^{[22]}$ and by visual inspection and selection of the tautomers that facilitate as many hydrogen bonds as possible. The histidine tautomeric forms considered in the calculations are all summarized in Table S1 of the Supporting Information (SI). Having assigned protonation states to the titratable residues using PROPKA 2.0, standard protonation states were assigned to all other residues. Subsequently, hydrogen atoms were added to the protein and water molecules using TINKER 5.0. ${ }^{[74]}$ The hydrogen atoms of the BV chromophore, in turn, were added manually using GaussView 5. ${ }^{[75]}$

At this point, the net charge of the complete system was -9 , including a chromophore net charge of -1 attributable to the combination of a cationic ${ }^{[76-79]}$ tetrapyrrole skeleton with two propionic side chains (Figure 2). To neutralize the system, nine sodium ions were introduced at $3 \AA$ distances from the carboxylate moieties of anionic aspartate and glutamate residues lacking other counterions and residing far away (> $16 \AA$ ) from the BV chromophore.

Standard $\mathrm{AMBER}^{[80]}$ force-field parameters were assigned to all amino acid residues except Cys24, which is covalently linked to the BV chromophore. Atomic charges of the chromophore and the side chain of Cys24 were derived from electrostaticpotential charges computed at the HF/6-31G(d) level of theory, which is consistent with the original development of the AMBER force field. For the main chain of Cys24, the atomic charges were assigned following the procedure of Morokuma and co-workers. ${ }^{[62]}$ Atom types of the chromophore were selected manually. Atom types and atomic charges of all non-standard groups included in the system are given in Tables S2 and S3 of the SI. The TIP3P model ${ }^{[81]}$ was invoked for the water molecules.

Using a two-layer ONIOM(QM:MM) ${ }^{[82]}$ scheme, including hydrogen link atoms, QM/MM calculations were carried out with the Gaussian 09 program. ${ }^{[83]}$ While the low layer was treated classically with the AMBER force field, the high layer was described 
using single-reference quantum chemical methods, and included the BV chromophore and the side chain of Cys24 unless otherwise noted (Figure 3). A larger QM system was also considered, as further described below. Through the inclusion of MM charges in the high-layer electronic Hamiltonian, the interaction between the two layers account for polarization of the high layer by the surrounding MM charges (so-called electronic embedding). ${ }^{[84]}$

For all QM/MM geometry optimizations, the $\mathrm{QM}$ system was treated with the B3LYP hybrid density functional in combination with, unless otherwise noted, the 6$31 \mathrm{G}(\mathrm{d}, \mathrm{p})$ basis set. First, all hydrogen atoms and sodium ions of the system were optimized using the 3-21G (rather than 6-31G(d,p)) basis set. Since this calculation left the positions of the protein heavy atoms unchanged, the resulting structure will henceforth be referred to as the crystallographic or experimental structure, and will be denoted $\mathrm{BV}_{\text {Exp }}$. Continuing from the experimental structure, a number of relaxed protein structures were obtained in the following way. First, keeping the protein environment fixed and relaxing to various degrees only the chromophore and Cys24, three additional structures were obtained in which only the bond lengths (structure denoted $\mathrm{BV}_{\mathrm{B}}$ ), only the bond lengths and bond angles $\left(\mathrm{BV}_{\mathrm{BA}}\right)$, and all geometric parameters of the chromophore and Cys24 (BV Full $)$ were allowed to vary, respectively. Second, two additional structures were optimized where, besides the chromophore and Cys24, either only the photochemically relevant Asp207 and His260 residues $\left(\mathrm{BV}_{\text {Full+D,H }}\right),{ }^{[19,20]}$ or all residues of the chromophore binding site ( $\left.\mathrm{BV}_{\text {Full+Site }}\right)$, were relaxed. Amino acid residues and water molecules of the chromophore binding site are listed in Table S4 of the SI.

\section{Calculation of UV-vis spectra}

Based on the various protein structures, vertical excitation energies were computed within the ONIOM(QM:MM) scheme by employing time-dependent density functional theory (TD-DFT ${ }^{[85-90]}$ for the QM system. From the resulting excitation energies, UVvis spectra were then obtained by convoluting 50-100 transitions with Gaussian functions of fixed full width at half maximum (set to $0.5 \mathrm{eV}$ ). 
The TD-DFT calculations were carried out with ten different density functionals covering a variety of exchange-correlation potentials, including BP86, BLYP, B97D ${ }^{\text {[91] }}$ (functionals based on the generalized gradient approximation [GGA]), $\tau$-HCTH ${ }^{[92]}$ (a meta-GGA), B3LYP, PBE0 (hybrid GGAs), M06-HF ${ }^{[93]}$ (a hybrid meta-GGA), LC-

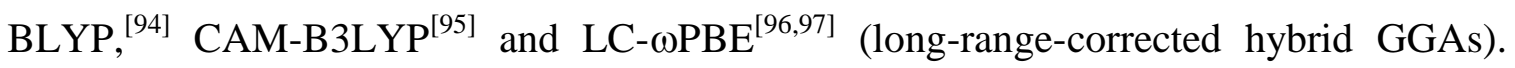
While BP86, BLYP, B3LYP and PBE0 are widely used standard functionals, $\tau$-HCTH includes a dependence on the kinetic-energy density. The reason for using the M06-HF, LC-BLYP, CAM-B3LYP and LC- $\omega$ PBE functionals, in turn, is that they have partly been developed with the aim to provide a better description of excited states than standard functionals, either by including full Hartree-Fock (exact) exchange or allowing the fraction of Hartree-Fock exchange to depend on the interelectronic distance. This particularly improves the description of excited states with charge-transfer character. ${ }^{[89,93-97]}$ Three different Pople-style basis sets were used for the TD-DFT calculations: 6-31G(d,p), 6-31+G(d,p) and 6-311G(2df,p).

As noted above, the default QM system for the QM/MM calculations contained the BV chromophore and the side chain of Cys24. However, to assess the convergence of the QM/MM excitation energies with respect to the size of the QM system, additional excited-state calculations were also performed using a larger QM system. This included also the highly conserved Asp207 and His260 residues, which are believed to play a key role for the successful completion of the $\mathrm{Pr} \rightarrow$ Pfr conversion. ${ }^{[19,20]}$ Furthermore, the larger QM system also included a water molecule (the so-called pyrrole water) positioned in close proximity to the A, B and C-ring nitrogens of the chromophore (Figure 3). Together with the chromophore, these groups form a hydrogen-bonding network whereby the negative partial charges of the Asp207 backbone carbonyl oxygen and the His260 $\delta 1$ nitrogen stabilize the cationic tetrapyrrole skeleton of the chromophore. ${ }^{[4,23]}$ For His260, only the imidazole side chain was included in the QM system. Henceforth, the default and expanded QM systems will be referred to as QM1 and QM2, respectively.

In previous studies, the UV-vis spectra of the bilin chromophores of phytochromes have been computed using an implicit reaction-field description of the surrounding protein. ${ }^{[49,50]}$ Although such an approach includes bulk electrostatic effects, it does not account for specific short-range chromophore-protein interactions. Thus, it is of 
interest to compare this approach with the present QM/MM-based methodology, which includes both bulk and short-range effects. Accordingly, complementary calculations investigating the absorption features of the $\mathrm{BV}$ chromophore in a dielectric continuum were carried out with the SMD continuum solvation model. ${ }^{[98]}$ Following Blomberg et al., ${ }^{\text {[99] }}$ a solvent (diethylether [ $\left.\mathrm{Et}_{2} \mathrm{O}\right]$ ) with a dielectric constant of around 4 (4.24) was chosen to represent the protein. These calculations were done with the $6-31+G(d, p)$ basis set.

\section{Results and Discussion}

\section{Performance of different density functionals}

In this section, we will present results summarized in Table 1 that show how the choice of density functional for the QM system affects the computed Q- and Soret-band absorption maxima. Since, at present, all bilin chromophores used by phytochromes are too large for routine calculations with high-level $a b$ initio methods rather than TD-DFT, these results constitute a valuable set of benchmark data for future QM/MM calculations on phytochrome proteins. The calculations were carried out based on the $\mathrm{BV}_{\text {FulltSite }}$ structure (see computational details) and used the default QM system (QM1) comprising the BV chromophore and the side chain of Cys24 (Figure 3). The basis set was 6$31+G(d, p)$, which includes diffuse functions on the heavy atoms. It should be pointed out that, for both bands, the value obtained by summing Gaussian-convoluted TD-DFT transition energies (shown in Table 1) is throughout similar to the energy (not shown) of the transition with the largest oscillator strength within the band. Specifically, the differences between the tabulated absorption maxima and the energies of the transitions with the largest oscillator strengths lie consistently within 0.03 (Q band) and $0.08 \mathrm{eV}$ (Soret band).

Experimentally, the Pr form of $\mathrm{DrBphP}$ has a Q band peaking around $700 \mathrm{~nm}$ $(1.77 \mathrm{eV}) .{ }^{[20]}$ Among the different density functionals considered in the calculations, the best agreement with this value is shown by the pure GGAs (BP86, BLYP, B97D and $\tau$ HCTH), which deviate by less than $0.15 \mathrm{eV}$. Notably, all functionals blue-shift the 
absorption maximum of the $\mathrm{Q}$ band relative to the experimental position, which is in accord with early QM studies of the tetrapyrrole porphin molecule. ${ }^{[85,100]}$ In light of the well-known tendency of pure GGAs to give too low excitation energies, ${ }^{[101]}$ however, the good performance of the pure GGAs should not necessarily be taken as an indication that they are intrinsically more accurate for the photochemical problem at hand than the exact-exchange-containing B3LYP and PBE0 hybrid GGAs, which deviate from the experimental value by about $0.3 \mathrm{eV}$.

It is further interesting to note that the functionals exhibiting the worst agreement $(\sim 0.5 \mathrm{eV})$ with the experimental Q-band maximum (M06-HF, LC-BLYP, CAM-B3LYP and LC- $\omega \mathrm{PBE}$ ) are actually those that, in part, have been designed to provide accurate excitation energies, particularly with respect to the inadequate treatment by standard functionals of excited states with strong charge-transfer character. ${ }^{[89,102]}$ It is plausible that this observation can be explained from the fact that we have not found any evidence from population analyses that charge-transfer excitations contribute to the $\mathrm{Q}$ band. Indeed, among the 50-100 transitions included in the Gaussian convolution, those exhibiting charge-transfer character have negligible oscillator strengths. In accord with previous studies, ${ }^{[89,102]}$ the M06-HF, LC-BLYP, CAM-B3LYP and LC- $\omega$ PBE functionals place the corresponding transitions at higher energies than do the standard functionals, although the effect is smaller than what was observed for the pure charge-transfer states considered therein.

As for the Soret band, the peak at around $380 \mathrm{~nm}(3.26 \mathrm{eV})$ exhibited by the $\mathrm{Pr}$ form of $\operatorname{DrBphP}^{[20]}$ is also better reproduced by the functionals not primarily aimed at excited-state studies. In fact, while M06-HF, LC-BLYP and LC- $\omega$ PBE blue-shift this absorption maximum by up to $0.5-0.6 \mathrm{eV}$ relative to the experimental value ( $\sim .3 \mathrm{eV}$ for CAM-B3LYP), all the other functionals perform well and deviate by no more than 0.13 $\mathrm{eV}$. In analogy with the calculations for the $\mathrm{Q}$ band, the absorption maxima obtained with the pure GGAs are red-shifted relative to those obtained with B3LYP and PBE0, but (unlike the calculations for the $\mathrm{Q}$ band) are also red-shifted relative to the experimental value.

In addition to using TD-DFT for the QM system, we have also tested the performance of configuration interaction singles (CIS) with perturbation-theory treatment 
of doubles corrections (CIS(D)). ${ }^{[103]}$ This method is the cheapest $a b$ initio approach for accounting for electron correlation in excited states, and usually offers a significant improvement over the standard CIS method, ${ }^{[45,103]}$ which neglects electron correlation. However, since it is challenging to consider 50-100 transitions even at this level of theory, the CIS(D) absorption maxima were obtained as the energies of the lowest $\left(\mathrm{S}_{1}, \mathrm{Q}\right.$ band) and second-lowest ( $\mathrm{S}_{2}$, Soret band) excited states dominating the two bands, which was found to be an adequate procedure at the less demanding TD-DFT and CIS levels. Furthermore, the CIS(D) calculations were done with the 6-31G(d,p) basis set instead of $6-31+G(d, p)$, thus omitting diffuse functions on the heavy atoms. The importance of diffuse functions will be assessed in the next section. From Table 1, it can be seen that CIS blue-shifts the absorption maxima of the $\mathrm{Q}$ and Soret bands by about $1 \mathrm{eV}$, which is a rather typical error for this method even in favorable cases. ${ }^{[104]}$ Adding the perturbative doubles corrections improves the results quite considerably (by about $0.5-0.6 \mathrm{eV}$ ), but is not sufficient to render them competitive with the results of the best-performing density functionals.

Having carried out $\mathrm{QM} / \mathrm{MM}$ calculations on the $\operatorname{DrBphP}$ protein-chromophore complex with a variety of density functionals, it is of interest to explore to what extent the differences in performance between the functionals are governed by the intrinsic accuracy they afford for the bare chromophore. To this end, complementary TD-DFT, CIS and CIS(D) calculations were executed to estimate the Q-band absorption maximum of the BV chromophore in the gas phase. In the absence of an experimental gas-phase spectrum, the reference value for these calculations was taken to be the corresponding absorption maximum obtained with the symmetry-adapted-cluster CI (SAC-CI) method, ${ }^{[105]}$ which together with, e.g., the spectroscopy-oriented CI method ${ }^{[106]}$ is one of few high-level $a b$ initio approaches applicable to the excited states of bilin chromophores. Given that static correlation effects are of minor importance at the equilibrium geometry of the singly excited $\mathrm{S}_{1}$ state, ${ }^{[43]} \mathrm{SAC}-\mathrm{CI}$ is expected to provide a gas-phase absorption maximum (here $S_{0} \rightarrow S_{1}$ excitation energy) of reasonable accuracy. As described in Table S5 of the SI and imposed by the computational cost of SAC-CI, all these calculations were done with a simplified BV chromophore model henceforth denoted BVmod. This model retains the tetrapyrrole skeleton of the full chromophore. 
Comparing the QM data summarized in Table S5 with the corresponding $\mathrm{QM} / \mathrm{MM}$ results in Table 1, it is clear that the QM/MM performance of the functionals largely mirrors the accuracy they afford for the bare chromophore. Indeed, the two series show exactly the same trends where: 1) all functionals blue-shift the respective absorption maximum relative the experimental or SAC-CI reference value; and 2) the pure GGAs (BP86, BLYP, B97D and $\tau$-HCTH) give smaller blue shifts than both the (global) hybrids that include a fixed fraction of exact exchange (B3LYP [20\%], PBE0 [25\%] and M06-HF [100\%]) and the long-range-corrected hybrids that allow the fraction of exact exchange to grow with the interelectronic distance (LC-BLYP, CAM-B3LYP and LC- $\omega \mathrm{PBE})$. For both series, it can also be seen that the absorption maxima obtained with the hybrid functionals, starting with B3LYP and PBE0, are increasingly blue-shifted as the fraction of exact exchange is incremented. This observation is in line with the extensive TD-DFT benchmark study of Adamo and co-workers. ${ }^{[101]}$ We will return to a more quantitative comparison of gas-phase QM and $\mathrm{DrBphP} \mathrm{QM} / \mathrm{MM}$ calculations in a later section.

\section{Size of the QM system}

All QM/MM calculations of the preceding section were carried out using the default QM system (QM1) comprising the BV chromophore and the side chain of Cys24 (Figure 3). From the viewpoint of the excited-state calculations, this is one of the smallest QM system that one can conceive, save the inclusion of the side chain of Cys24 and the C8 and C12 propionic substituents of the chromophore. While choosing such a QM system is advantageous in terms of computational effort, it is also of interest to investigate whether the estimates of the Q- and Soret-band absorption maxima are sensitive to enlargement of the QM system. Accordingly, additional QM/MM excited-state calculations were executed using the expanded QM system (QM2) containing also the highly conserved Asp207 and His260 residues and the pyrrole water (Figure 3). As noted above, these groups form a hydrogen-bonding network with the chromophore and are thought to play a key role for the successful completion of the $\operatorname{Pr} \rightarrow \operatorname{Pfr}$ conversion. ${ }^{[19,20]}$ As before, the 
calculations were based on the $\mathrm{BV}_{\text {Full+Site }}$ structure and were done with the 6-31+G(d,p) basis set. The results are given in Table 2.

Considering first the $\mathrm{Q}$ band, the excitation of which underlies the primary photochemistry of phytochromes, we note that including Asp207 and His260 in the QM system does not seem worthwhile in light of the added computational cost and the relatively minor improvement with respect to the QM1 values. Indeed, treating these residues quantum mechanically rather than classically reduces the blue shift relative to the experimental absorption maximum at $1.77 \mathrm{eV}^{[20]}$ by

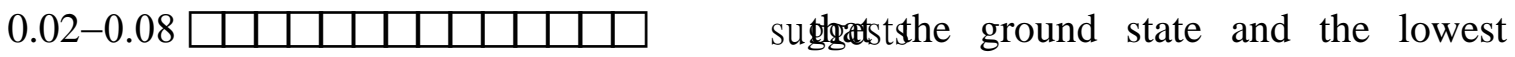
excited state dominating the $\mathrm{Q}$ band derive similar stabilization from the hydrogenbonding network, and that future QM/MM calculations aimed at deciphering the detailed $\operatorname{Pr} \rightarrow$ Lumi-R photoisomerization mechanism (which is a key question in phytochrome research $^{[15-17]}$ ) can be carried out with a small QM system. As for the Soret band, the situation is completely analogous. Specifically, for most methods, enlarging the QM system red-shifts the absorption maximum by $\sim 0.05$ eV only.

Besides using the $6-31+G(d, p)$ basis set for the excited-state calculations, complementary calculations were also done with two other basis sets: 6-31G(d,p) and 6311G(2df,p). These calculations are summarized in Table 3, and made use of the $\mathrm{BV}_{\text {Full+Site }}$ structure and the default QM system (QM1). The reason for probing the effect of diffuse functions $(6-31 G(d, p)$ vs. $6-31+G(d, p))$ is their tentative importance for properly describing the anionic chromophore, whereas the comparison between the 631G(d,p) and 6-311G(2df,p) basis sets probes whether additional polarization functions are needed.

For the $\mathrm{Q}$ band, the data in Table 3 show that the diffuse functions of the 6$31+\mathrm{G}(\mathrm{d}, \mathrm{p})$ basis set reduce the blue shift relative to the experimental absorption maximum at $1.77 \mathrm{eV},{ }^{[20]}$ but that the improvement is small $(0.03-0.04 \mathrm{eV})$ for all methods considered. Hence, the use of diffuse functions for this band appears not to be particularly important, which we believe is a consequence of the fact that the transitions (1 contributingephedingstoto the batidlodide localized on the cationic tetrapyrrole moiety. Further, it can be seen that including polarization functions beyond those already included in the $6-31+G(d, p)$ basis set is not worthwhile, 
as the 6-311G(2df,p) results for the $\mathrm{Q}$ band are only marginally $(0.02-0.03 \mathrm{eV})$ better than the 6-31G(d,p) results. The basis-set effects for the Soret band, in turn, follow a similar pattern, although diffuse functions give slightly larger red shifts for this band $(0.05-0.11 \mathrm{eV})$ than for the $\mathrm{Q}$ band $(0.03-0.04 \mathrm{eV})$.

\section{Influence of structural relaxation}

Before discussing how structural relaxation influences the calculated absorption maxima, it is worthwhile to point out one interesting feature of the $\mathrm{BV}_{\text {Full }+ \text { Site }}$ structure, in which the BV chromophore as well as all amino acid residues and water molecules of the chromophore binding site have been fully relaxed. Namely, this structure shows a significant out-of-plane orientation of the $\mathrm{D}$ ring relative to the other pyrrole rings of the chromophore. Specifically, as detailed in Table S6 of the SI, the C13-C14-C16-N(D) twist angle between the $\mathrm{C}$ and $\mathrm{D}$ rings is much larger $\left(36^{\circ}\right)$ than the corresponding angles between the $\mathrm{A}$ and $\mathrm{B}\left(18^{\circ}\right)$ and $\mathrm{B}$ and $\mathrm{C}\left(-1^{\circ}\right)$ rings. Similar twist angles are also shown by the $\mathrm{DrBphP}$ crystal structure upon which the calculations are based. ${ }^{[23]}$ Since, as noted above, it is commonly accepted that the $Z \rightarrow E$ photoisomerization that initiates the $\operatorname{Pr} \rightarrow$ Pfr conversion occurs at the C15-C16 bond of the methine bridge between the $\mathrm{C}$ and $\mathrm{D}$ rings (Figure 2), ${ }^{[13-15,28,30,32,33]}$ such pre-twisting is likely to facilitate this reaction, which can also be inferred from the actual methine bridge dihedral angles of the DrBphP crystal

structure (Table S6). ${ }^{[23]}$ Indeed, according to a recent estimate, ${ }^{[15]}$ the isomerization occurs within 3 ps and produces the so-called Lumi-R intermediate within a few tens of ps after light absorption. ${ }^{[4,35]}$ Notably, however, pre-twisting of the C15-C16 bond is not a conserved feature among all phytochrome crystal structures reported to date. ${ }^{[25]}$

All absorption data reported up to this point were derived from the $\mathrm{BV}_{\text {Full+Site }}$ structure just discussed. However, it is also of interest to consider alternative structures that "fall between" this structure and the crystallographic $B V_{\operatorname{Exp}}$ structure with respect to the degree of relaxation. This holds true in particular since recent QM-based studies of bare bilin chromophores using an implicit reaction-field representation of the protein environment have shown better agreement with experimental absorption data when the calculations are performed on crystallographic rather than relaxed chromophore 
structures. ${ }^{[49,50]}$ To this end, the absorption maxima of the Q and Soret bands were also computed based on the $\mathrm{BV}_{\text {Exp }}, \mathrm{BV}_{\mathrm{B}}, \mathrm{BV}_{\mathrm{BA}}, \mathrm{BV}_{\text {Full }}$ and $\mathrm{BV}_{\text {Full+D,H }}$ protein structures (see computational details). The results of these calculations, which were done with the expanded QM system (QM2) and the 6-31+G(d,p) basis set, are given in Figures 4 and 5.

Focusing the discussion on the Q band (Figure 4), the calculated absorption maxima exhibit clear convergence as the crystallographic $\mathrm{BV}_{\operatorname{Exp}}$ structure is transformed into the $\mathrm{BV}_{\text {Full }+ \text { Site }}$ structure by first allowing the chromophore and Cys 24 to fully relax $\left(\mathrm{BV}_{\mathrm{Exp}} \rightarrow \mathrm{BV}_{\mathrm{B}} \rightarrow \mathrm{BV}_{\mathrm{BA}} \rightarrow \mathrm{BV}_{\text {Full }}\right.$ ), and subsequently also allowing for relaxation of the Asp207 and His260 residues and the entire chromophore binding site $\left(\mathrm{BV}_{\text {Full }} \rightarrow\right.$ $\left.\mathrm{BV}_{\text {Full }+\mathrm{D}, \mathrm{H}} \rightarrow \mathrm{BV}_{\text {Full }+ \text { Site }}\right)$. However, the overall best agreement with the experimental Qband maximum at $1.77 \mathrm{eV}^{[20]}$ is obtained using the $\mathrm{BV}_{\operatorname{Exp}}$ structure, for which all functionals are accurate to within $\sim 0.2 \mathrm{eV}$ or better. Although a similar observation was made by Matute et al. ${ }^{[49,50]}$ in the aforementioned QM-based studies, it is clear that it is because of cancellation of errors that the crystallographic structure yields better results than the relaxed protein structures, where the chromophore geometry has been refined quantum mechanically. Support for this view can be found in a number of earlier computational studies of protein-cofactor systems. ${ }^{[68,107-109]}$

From Figure 4, it can be seen that optimizing the bond lengths of the chromophore and Cys24 $\left(\mathrm{BV}_{\mathrm{Exp}} \rightarrow \mathrm{BV}_{\mathrm{B}}\right)$ blue-shifts the absorption maxima by $\sim 0.1 \mathrm{eV}$ compared to the values associated with the crystallographic structure. The most notable difference between the bond lengths of the $\mathrm{BV}_{\operatorname{Exp}}$ and $\mathrm{BV}_{\mathrm{B}}$ structures concerns the C14-C15 and $\mathrm{C} 15-\mathrm{C} 16$ bonds of the $\mathrm{CD}$ methine bridge (Figure 2). In the $\mathrm{BV}_{\operatorname{Exp}}$ structure, C14-C15 has double-bond character and C15-C16 has single-bond character. It is, however, straightforward to show that the only chromophore resonance structures compatible with the finding that the positive charge of the tetrapyrrole skeleton resides predominantly at the B and C rings, ${ }^{[45,46,110,111]}$ are those where, conversely, C14-C15 has single-bond character and C15-C16 has double-bond character. This is indeed the case in $\mathrm{BV}_{\mathrm{B}}$. Optimizing also the bond angles $\left(\mathrm{BV}_{\mathrm{B}} \rightarrow \mathrm{BV}_{\mathrm{BA}}\right)$ does not affect the absorption maxima. Torsional relaxation $\left(\mathrm{BV}_{\mathrm{BA}} \rightarrow \mathrm{BV}_{\text {Full }}\right)$, on the other hand, results in an additional $\sim 0.1 \mathrm{eV}$ blue shift, which can be understood in terms of the sensitivity of the 
frontier $\pi$-orbitals - that are responsible for the $\mathrm{Q}$ band ${ }^{[112]}$ - to the relative orientations of the four pyrrole rings. Finally, we note that relaxation of the amino acid residues and water molecules of the chromophore binding site ( $\left.\mathrm{BV}_{\text {Full }} \rightarrow \mathrm{BV}_{\text {Full+D,H }} \rightarrow \mathrm{BV}_{\text {Full+Site }}\right)$ leaves the absorption maxima unchanged. As for the water molecules, this finding reflects that they are dislocated no more than $\sim 0.1 \AA$ from their crystallographic positions during the optimization, as further detailed in Table S7 of the SI.

Having discussed structural relaxation effects, it is also of interest to assess how the use of another method than B3LYP for the QM part of the QM/MM geometry optimizations would affect the calculated absorption maxima. For example, in a related study of rhodopsin proteins, it was found that excitation energies of the retinal chromophore are very sensitive to the bond length alternation (BLA) in the conjugated polyene chain, and thereby to the method used for geometry optimization. ${ }^{[113]}$ To explore this issue, a series of QM calculations were carried out where first the geometry of the BV chromophore was optimized with B3LYP, BLYP, HF, MP2 and, using the TURBOMOLE 6.3 program, ${ }^{[114,115]}$ the approximate coupled-cluster singles and doubles (CC2) method. ${ }^{[116]}$ Then, based on the resulting geometries, Q-band absorption maxima were computed employing TD-DFT with the same set of functionals considered in the QM/MM calculations. The results of this investigation are collected in Tables S8 and S9 of the SI.

Exemplifying the BLA through the $\mathrm{C}-\mathrm{C}$ bonds of the three methine bridges and noting that the C9-C10 and C10-C11 bonds are near-identical because of resonance stabilization, ${ }^{[42-44]}$ Table S8 shows that the pattern predicted by B3LYP for these six bonds agrees to within $0.005 \AA$ with the MP2 pattern, but differs somewhat more from the patterns obtained by CC2 (by up to $0.011 \AA$ ) and BLYP $(0.014 \AA$ ), which favor less BLA. The difference with respect to HF, in turn, is substantial (by up to $0.064 \AA$ ) and in line with the well-known tendency of HF to overestimate the BLA in conjugated systems. Accordingly, as for the spectral effect (Table S9), using the MP2, CC2 or BLYP geometry instead of the B3LYP geometry shifts the absorption maxima much less than what using HF does: 0.02-0.04 (MP2), 0.05-0.08 (CC2), 0.06-0.09 (BLYP) and 0.06-0.37 eV (HF). The relative similarity between B3LYP, MP2, CC2 and BLYP in this regard, whereby the BV chromophore seems to pose a lesser computational challenge 
than, e.g., retinal, ${ }^{[113]}$ is an indication that the exclusive use of B3LYP for all QM/MM geometry optimizations is not a factor that strongly limits the scope of this work.

\section{Implications for spectral tuning}

The results from the calculations comparing the present QM/MM-based approach with one where the protein is described using the SMD continuum solvation model ${ }^{[98]}$ are presented in Table 4, which focuses on the Q band. The corresponding results for the Soret band are given in Table S10 of the SI. In these calculations, the BV chromophore was first cut out from the $\mathrm{BV}_{\text {Full }+ \text { Site }}$ structure, and then its absorption maxima were computed for this very geometry, except that the propionic side chains were replaced by hydrogen atoms. In earlier studies, these substitutions have been shown to have a negligible effect on the light absorption of bilin chromophores. ${ }^{[40]}$ In addition to calculations with the SMD model, the absorption maxima were also computed in the gas phase.

Starting with the SMD calculations in Table 4, the resulting absorption maxima $\left(\lambda_{\max }{ }^{(3)}\right)$ are red-shifted relative to their QM/MM counterparts $\left(\lambda_{\max }{ }^{(4)}\right)$ by $0.15-0.19 \mathrm{eV}$. This suggests that the sum of all specific short-range chromophore-protein interactions not accounted for by the SMD model but included in the QM/MM methodology overall blue-shift the $\mathrm{Q}$ band $\left(\lambda_{\max }{ }^{(3)} \rightarrow \lambda_{\max }{ }^{(4)}\right)$. Importantly, this does not mean that all such interactions, which would seem necessary for chromophore binding, individually exert a blue-shifting effect; rather, it indicates that their combined effect (of blue-shifting and red-shifting interactions) is a blue shift. Continuing with the gas-phase calculations on the protein geometry, the corresponding absorption maxima $\left(\lambda_{\max }{ }^{(2)}\right)$ are blue-shifted relative to the SMD values by $0.07-0.19 \mathrm{eV}$. This, in turn, suggests that the long-range bulk electrostatic effects from the protein included in the SMD model red-shift the Q band $\left(\lambda_{\max }{ }^{(2)} \rightarrow \lambda_{\max }{ }^{(3)}\right)$.

Qualitatively, the short-range blue-shifting and long-range red-shifting effects appear to be of comparable magnitudes, because the gas-phase and QM/MM absorption maxima are quite similar at the protein geometry. However, for the pure GGAs the gasphase maxima are slightly blue-shifted with respect to their positions in the protein, 
whereas for the hybrid functionals (both global and long-range-corrected) they are redshifted. Thus, it is desirable to assess which of these scenarios is more accurate. Unfortunately, in the absence of an experimental gas-phase absorption spectrum with which the $\operatorname{DrBphP}$ Q-band peak at $1.77 \mathrm{eV}^{[20]}$ can be compared, there is no experimental information on this shift. Instead, the $\operatorname{DrBphP}$ Q-band peak was compared with a theoretical estimate of the gas-phase absorption maximum derived by first calculating, at the geometry shown by the $\mathrm{BV}_{\text {Full }+ \text { Site }}$ structure, the $\mathrm{S}_{0} \rightarrow \mathrm{S}_{1}$ excitation energy of the previously discussed (Table S5) BVmod chromophore model using SAC-CI/6-31G(d,p) $(=1.70 \mathrm{eV})$, and subsequently calculating how this energy would change in the presence of the missing chromophore substituents using CIS(D)/6-31G(d,p) $(=-0.05 \mathrm{eV})$.

Based on these calculations, it was estimated that the protein environment blueshifts the intrinsic gas-phase absorption by $1.77-1.65=0.12 \mathrm{eV}$. In light of the finding that $\mathrm{QM} / \mathrm{MM}$ calculations with pure GGAs give the best performance relative to the experimental $\operatorname{DrBphP}$ Q-band peak, it is then notable that it is actually the hybrid functionals that most closely reproduce this shift. Indeed, the $\lambda_{\max }{ }^{(2)} \rightarrow \lambda_{\max }{ }^{(4)}$ values for these methods correspond to blue shifts of $0.08-0.11 \mathrm{eV}$ and also match the CIS(D) value of $0.14 \mathrm{eV}$, whereas the pure GGAs give red shifts of $0.03-0.04 \mathrm{eV}$. In this regard, an earlier computational study of spectral tuning in rhodopsin proteins found that TD-DFT methods are not able to fully account for absorption shifts induced by an electrostatic environment defined, just as in the current $\mathrm{QM} / \mathrm{MM}$ scheme, in terms of atomic point charges. ${ }^{[113]}$ While a shift of the order of $0.1 \mathrm{eV}$ is likely too small to allow for a more detailed analysis along those lines, the observation that the effect for rhodopsins is more prominent using pure GGAs than hybrid functionals ${ }^{[113]}$ is possibly reflected in the differences that we here observe in the corresponding shifts for $\operatorname{DrBphP}$.

Besides spectral tuning by the electrostatic environment, the protein also perturbs the geometry of the chromophore, which may further alter the absorption. To quantify this effect, the same exact $a b$ initio approach undertaken above to obtain a putatively accurate gas-phase absorption maximum of the chromophore its protein-bound geometry, was followed in additional SAC-CI and CIS(D) calculations at the gas-phase B3LYP/6$31 \mathrm{G}(\mathrm{d}, \mathrm{p})$ geometry. In this way, it was estimated that the protein-induced change in chromophore geometry blue-shifts the absorption by $0.10 \mathrm{eV}$. Adding this result to the 
$0.12 \mathrm{eV}$ blue shift attributed to the "non-geometric" perturbations, it is then predicted that the total net effect of the protein is to blue-shift the $\mathrm{Q}$ band by about $0.2 \mathrm{eV}$. Returning to the calculations in Table 4 , the $\lambda_{\max }{ }^{(1)} \rightarrow \lambda_{\max }{ }^{(2)}$ values show that all functionals associate the geometric perturbation with a blue shift of $0.09-0.17 \mathrm{eV}$, which is close to the SACCI-extrapolated result of $0.10 \mathrm{eV}$. Adding these shifts to the non-geometric $\lambda_{\max }{ }^{(2)} \rightarrow$ $\lambda_{\max }{ }^{(4)}$ shifts, one then obtains estimates of the total protein-induced effect $\left(\lambda_{\max }{ }^{(1)} \rightarrow\right.$ $\left.\lambda_{\max }{ }^{(4)}\right)$ that range from a $0.06-0.07 \mathrm{eV}$ blue shift for the pure GGAs to a $0.19-0.28 \mathrm{eV}$ blue shift for the hybrid functionals, which again exhibit better agreement with the reference value $(\sim 0.2 \mathrm{eV})$.

Finally, it is notable that the reaction-field approach $\left(\lambda_{\max }{ }^{(3)}\right)$ is competitive with the more demanding QM/MM approach $\left(\lambda_{\max }{ }^{(4)}\right)$ in terms of reproducing the experimental Q-band maximum at $1.77 \mathrm{eV} .{ }^{[20]}$ Indeed, the SMD absorption maxima obtained with the best-performing functionals (BP86, BLYP, B97D and $\tau$-HCTH) agree very well with the experimental value, although cancellation of errors clearly contributes to this result.

\section{Conclusions}

In summary, we have reported a study of how QM/MM methods are best employed to compute the Q- and Soret-band absorption maxima of phytochromes, which constitute one of the least well-characterized families of photosensory proteins with regard to computational studies. Specifically, identifying the Pr form of $\mathrm{DrBphP}$ as a suitable system for such an investigation and starting from a high-resolution (1.45 $\AA$ ) crystal structure thereof, ${ }^{[23]}$ we have carried out $\mathrm{ONIOM}(\mathrm{QM}: \mathrm{MM})$ electronic-embedding calculations using TD-DFT for the QM system and the AMBER force field for the MM system, to explore what factors govern the accuracy of the resulting absorption maxima. Thereby, we have also gained valuable new insight into the effect of the protein environment on the light absorption of the BV chromophore.

Overall, we believe that our study serves as a useful benchmark for future QM/MM studies of photochemical problems in the field of phytochrome research. At the same time, further benchmarks are needed to explore some of the methodological issues 
that we have not addressed in this work, such as the merits of performing molecular dynamics simulations to both relax the protein structure and account for the effect of thermal motion on the computed absorption spectra. In a different context of relevance for understanding the photosynthetic excitation energy transfer across the so-called Fenna-Matthews-Olson complex found in green sulfur bacteria, ${ }^{[117]}$ which contain bilinrelated (bacteriochlorophyll) chromophores, such effects have recently been incorporated in computational studies using a somewhat less demanding QM description than we have used in the present work. ${ }^{[118,119]}$

The conclusions of our study can be summarized as follows. First, testing the accuracy of a variety of density functionals for the QM system, it is found that the pure GGAs (BP86, BLYP, B97D and $\tau-\mathrm{HCTH}$ ) reproduce the experimental Q-band maximum $^{[20]}$ in the red region with smaller errors $(<0.15 \mathrm{eV})$ than both the B3LYP, PBE0 and M06-HF global hybrids that contain a fixed amount of exact exchange $(\sim 0.3-0.5 \mathrm{eV})$, and the LC-BLYP, CAM-B3LYP and LC- $\omega \mathrm{PBE}$ long-range-corrected hybrids for which the amount of exact exchange varies with the interelectronic distance $(\sim 0.5 \mathrm{eV})$. Relative to the experimental value, all methods blue-shift the absorption peak of this band, which is responsible for the primary photochemistry of phytochromes. For the hybrid functionals, the error increases with the amount of exact exchange included. Furthermore, from complementary QM-only calculations, it is inferred that the QM/MM performance of the functionals is largely governed by the accuracy afforded for the bare chromophore. As for the Soret band, in turn, M06-HF, LC-BLYP, CAM-B3LYP and LC$\omega \mathrm{PBE}$ continue to perform less well than the other methods, which all reproduce the corresponding experimental peak ${ }^{[20]}$ in the blue region with errors smaller than $0.15 \mathrm{eV}$.

Second, investigating how the size of the QM system affects the computed absorption maxima, it is found that the results just mentioned obtained for a QM system comprising the chromophore and Cys24 side chain (to which the chromophore is attached) are similar to corresponding results obtained using a larger QM system including also the components (Asp207, His260 and the pyrrole water molecule) of the hydrogen-bonding network that stabilizes the chromophore in the protein. ${ }^{[4,23]}$ Specifically, with the enlarged QM system the blue shifts of the computed Q-band maxima are reduced, but only by $0.02-0.08 \square$ A comparable effect is documented for 
the Soret band. $\square \square \square \square \square$ suggests thafuture QM/MM studies of the primary photochemistry of phytochromes can be carried out with a small QM system.

Third, exploring the influence of structural relaxation, it is observed that the computed absorption maxima converge as further and further geometric parameters of the original crystal structure ${ }^{[23]}$ are relaxed, starting with the bond lengths of the chromophore and Cys24 and concluding with all parameters of the entire chromophore binding site. In fact, convergence is reached once the chromophore and Cys24 are fully relaxed. As for the agreement with the experimental absorption maxima, ${ }^{[20]}$ however, the Q band is overall better reproduced using the crystallographic structure than any of the relaxed structures. Indeed, for the former, all functionals give a Q-band peak to within $\sim 0.2 \mathrm{eV}$ or less of the experimental value, with subsequent geometry optimizations of the chromophore and Cys24 blue-shifting the results by 0.1 (relaxation of bond lengths only) and $\sim 0.2 \mathrm{eV}$ (full relaxation).

Fourth, comparing the QM/MM results with chromophore absorption maxima calculated both in the gas phase and with an implicit reaction-field description of the protein, it is suggested that the sum of all specific short-range chromophore-protein interactions blue-shift the $\mathrm{Q}$ band, whereas, conversely, long-range bulk electrostatic effects red-shift it. Moreover, obtaining a measure of the (experimentally unknown) net protein-induced absorption shift by taking the difference between the experimental peak maximum of $\operatorname{DrBphP}^{[20]}$ and a SAC-CI-extrapolated peak maximum of the bare chromophore at its protein-bound geometry, it is estimated that the protein environment, excluding its effect on the chromophore geometry, overall blue-shifts the intrinsic gasphase absorption of the $\mathrm{Q}$ band by about $0.1 \mathrm{eV}$. Interestingly, despite the fact that the pure GGAs offer better agreement with the protein absorption maximum, this shift is more accurately reproduced by the hybrid functionals (blue shifts of $0.08-0.11 \mathrm{eV}$ ) than by the pure GGAs, which actually predict red shifts of $0.03-0.04 \mathrm{eV}$. Comparing SACCI-extrapolated peak maxima of the bare chromophore at protein-bound and gas-phase geometries, it is further estimated that an additional blue shift of around $0.1 \mathrm{eV}$ can be attributed to the change in chromophore geometry in the protein. This result is also supported by TD-DFT-based calculations (blue shifts of 0.09-0.17 eV), and suggests that the total net effect of the protein is to blue-shift the $\mathrm{Q}$ band by about $0.2 \mathrm{eV}$. Finally, it is 
found that also for the total net effect do the hybrid functionals (blue shifts of 0.19-0.28 $\mathrm{eV}$ ) perform better than the pure GGAs (blue shifts of $0.06-0.07 \mathrm{eV}$ ).

\section{Acknowledgments}

B.D. gratefully acknowledges funding from Linköping University, the Swedish Research Council and the Olle Engkvist Foundation, and grants of computing time from the National Supercomputer Centre (NSC) in Linköping. 


\section{References}

[1] W. Rüdiger, F. Thümmler, Angew. Chem. Int. Ed. Engl. 1991, 30, 1216-1228.

[2] M. A. van der Horst, K. J. Hellingwerf, Acc. Chem. Res. 2004, 37, 13-20.

[3] B. Karniol, J. R. Wagner, J. M. Walker, R. D. Vierstra, Biochem. J. 2005, 392, 103-116.

[4] N. C. Rockwell, Y.-S. Su, J. C. Lagarias, Annu. Rev. Plant Biol. 2006, 57, 837-857.

[5] B. L. Montgomery, Mol. Microbiol. 2007, 64, 16-27.

[6] N. C. Rockwell, J. C. Lagarias, ChemPhysChem 2010, 11, 1172-1180.

[7] K. A. Franklin, P. H. Quail, J. Exp. Bot. 2010, 61, 11-24.

[8] A. Nagatani, Curr. Opin. Plant Biol. 2010, 13, 565-570.

[9] A. Möglich, X. Yang, R. A. Ayers, K. Moffat, Annu. Rev. Plant Biol. 2010, 61, 21-47.

[10] M. E. Auldridge, K. T. Forest, Crit. Rev. Biochem. Mol. Biol. 2011, 46, 67-88.

[11] A. T. Ulijasz, R. D. Vierstra, Curr. Opin. Plant Biol. 2011, 14, 498-506.

[12] V. A. Sineshchekov, Biochim. Biophys. Acta 1995, 1228, 125-164.

[13] J. C. Lagarias, H. Rapoport, J. Am. Chem. Soc. 1980, 102, 4821-4828.

[14] W. Rüdiger, F. Thümmler, E. Cmiel, S. Schneider, Proc. Natl. Acad. Sci. U.S.A. 1983, 80, 6244-6248.

[15] J. Dasgupta, R. R. Frontiera, K. C. Taylor, J. C. Lagarias, R. A. Mathies, Proc. Natl. Acad. Sci. U.S.A. 2009, 106, 1784-1789.

[16] K. M. Spillane, J. Dasgupta, R. A. Mathies, Biophys. J. 2012, 102, 709-717.

[17] Y. Yang, M. Linke, T. von Haimberger, J. Hahn, R. Matute, L. González, P. Schmieder, K. Heyne, J. Am. Chem. Soc. 2012, 134, 1408-1411.

[18] A. J. Fischer, J. C. Lagarias, Proc. Natl. Acad. Sci. U.S.A. 2004, 101, 17334-17339.

[19] D. von Stetten, S. Seibeck, N. Michael, P. Scheerer, M. A. Mroginski, D. H. Murgida, N. Krauss, M. P. Heyn, P. Hildebrandt, B. Borucki, T. Lamparter, J. Biol. Chem. 2007, 282, 2116-2123.

[20] J. R. Wagner, J. Zhang, D. von Stetten, M. Günther, D. H. Murgida, M. A. Mroginski, J. M. Walker, K. T. Forest, P. Hildebrandt, R. D. Vierstra, J. Biol. Chem. 2008, 283, 12212-12226. 
[21] J. Mailliet, G. Psakis, K. Feilke, V. Sineshchekov, L.-O. Essen, J. Hughes, J. Mol. Biol. 2011, 413, 115-127.

[22] J. R. Wagner, J. S. Brunzelle, K. T. Forest, R. D. Vierstra, Nature 2005, 438, 325-331.

[23] J. R. Wagner, J. Zhang, J. S. Brunzelle, R. D. Vierstra, K. T. Forest, J. Biol. Chem. 2007, 282, 12298-12309.

[24] X. Yang, E. A. Stojković, J. Kuk, K. Moffat, Proc. Natl. Acad. Sci. U.S.A. 2007, 104, 12571-12576.

[25] L.-O. Essen, J. Mailliet, J. Hughes, Proc. Natl. Acad. Sci. U.S.A. 2008, 105, $14709-14714$.

[26] X. Yang, J. Kuk, K. Moffat, Proc. Natl. Acad. Sci. U.S.A. 2008, 105, 14715-14720.

[27] X. Yang, J. Kuk, K. Moffat, Proc. Natl. Acad. Sci. U.S.A. 2009, 106, 15639-15644.

[28] X. Yang, Z. Ren, J. Kuk, K. Moffat, Nature 2011, 479, 428-432.

[29] J. Matysik, P. Hildebrandt, W. Schlamann, S. E. Braslavsky, K. Schaffner, Biochemistry 1995, 34, 10497-10507.

[30] F. Andel III, J. C. Lagarias, R. A. Mathies, Biochemistry 1996, 35, 15997-16008.

[31] H. Foerstendorf, E. Mummert, E. Schäfer, H. Scheer, F. Siebert, Biochemistry 1996, 35, 10793-10799.

[32] C. Kneip, P. Hildebrandt, W. Schlamann, S. E. Braslavsky, F. Mark, K. Schaffner, Biochemistry 1999, 38, 15185-15192.

[33] F. Andel III, J. T. Murphy, J. A. Haas, M. T. McDowell, I. van der Hoef, J. Lugtenburg, J. C. Lagarias, R. A. Mathies, Biochemistry 2000, 39, 2667-2676.

[34] K. Heyne, J. Herbst, D. Stehlik, B. Esteban, T. Lamparter, J. Hughes, R. Diller, Biophys. J. 2002, 82, 1004-1016.

[35] C. Schumann, R. Groß, N. Michael, T. Lamparter, R. Diller, ChemPhysChem 2007, $8,1657-1663$.

[36] J. J. van Thor, K. L. Ronayne, M. Towrie, J. Am. Chem. Soc. 2007, 129, 126-132.

[37] G. Cornilescu, A. T. Ulijasz, C. C. Cornilescu, J. L. Markley, R. D. Vierstra, J. Mol. Biol. 2008, 383, 403-413. 
[38] T. Rohmer, C. Lang, J. Hughes, L.-O. Essen, W. Gärtner, J. Matysik, Proc. Natl. Acad. Sci. U.S.A. 2008, 105, 15229-15234.

[39] A. T. Ulijasz, G. Cornilescu, C. C. Cornilescu, J. Zhang, M. Rivera, J. L. Markley, R. D. Vierstra, Nature 2010, 463, 250-256.

[40] B. Durbeej, O. A. Borg, L. A. Eriksson, Phys. Chem. Chem. Phys. 2004, 6, 5066-5073.

[41] B. Durbeej, O. A. Borg, L. A. Eriksson, Chem. Phys. Lett. 2005, 416, 83-88.

[42] P. Altoè, T. Climent, G. C. De Fusco, M. Stenta, A. Bottoni, L. Serrano-Andrés, M. Merchán, G. Orlandi, M. Garavelli, J. Phys. Chem. B 2009, 113, 15067-15073.

[43] B. Durbeej, Phys. Chem. Chem. Phys. 2009, 11, 1354-1361.

[44] A. Strambi, B. Durbeej, Photochem. Photobiol. Sci. 2011, 10, 569-579.

[45] O. A. Borg, B. Durbeej, J. Phys. Chem. B 2007, 111, 11554-11565.

[46] O. A. Borg, B. Durbeej, Phys. Chem. Chem. Phys. 2008, 10, 2528-2537.

[47] A. H. Göller, D. Strehlow, G. Hermann, ChemPhysChem 2005, 6, 1259-1268.

[48] J. Hasegawa, M. Isshiki, K. Fujimoto, H. Nakatsuji, Chem. Phys. Lett. 2005, 410, 90-93.

[49] R. A. Matute, R. Contreras, G. Pérez-Hernández, L. González, J. Phys. Chem. B 2008, 112, 16253-16256.

[50] R. A. Matute, R. Contreras, L. González, J. Phys. Chem. Lett. 2010, 1, 796-801.

[51] R. A. Friesner, V. Guallar, Annu. Rev. Phys. Chem. 2005, 56, 389-427.

[52] H. M. Senn, W. Thiel, Angew. Chem. Int. Ed. 2009, 48, 1198-1229.

[53] L. W. Chung, H. Hirao, X. Li, K. Morokuma, WIREs Comput. Mol. Sci. 2012, 2, 327-350.

[54] H. Hossein-Nejad, C. Curutchet, A. Kubica, G. D. Scholes, J. Phys. Chem. B 2011, 115, 5243-5253.

[55] C. Curutchet, J. Kongsted, A. Muñoz-Losa, H. Hossein-Nejad, G. D. Scholes, B. Mennucci, J. Am. Chem. Soc. 2011, 133, 3078-3084.

[56] C. Curutchet, V. I. Novoderezhkin, J. Kongsted, A. Muñoz-Losa, R. van Grondelle, G. D. Scholes, B. Mennucci, J. Phys. Chem. B 2012, DOI: 10.1021/jp305033d. 
[57] M. A. Mroginski, F. Mark, W. Thiel, P. Hildebrandt, Biophys. J. 2007, 93, 1885-1894.

[58] M. A. Mroginski, S. Kaminski, D. von Stetten, S. Ringsdorf, W. Gärtner, L.-O. Essen, P. Hildebrandt, J. Phys. Chem. B 2011, 115, 1220-1231.

[59] M. Hoffmann, M. Wanko, P. Strodel, P. H. König, T. Frauenheim, K. Schulten, W. Thiel, E. Tajkhorshid, M. Elstner, J. Am. Chem. Soc. 2006, 128, 10808-10818.

[60] P. B. Coto, A. Strambi, N. Ferré, M. Olivucci, Proc. Natl. Acad. Sci. U.S.A. 2006, 103, 17154-17159.

[61] S. Sekharan, M. Sugihara, V. Buss, Angew. Chem. Int. Ed. 2007, 46, 269-271.

[62] A. Altun, S. Yokoyama, K. Morokuma, J. Chem. Phys. B 2008, 112, 6814-6827.

[63] G. Tomasello, G. Olaso-González, P. Altoè, M. Stenta, L. Serrano-Andrés, M. Merchán, G. Orlandi, A. Bottoni, M. Garavelli, J. Am. Chem. Soc. 2009, 131, 5172-5186. [64] P. B. Coto, S. Martí, M. Oliva, M. Olivucci, M. Merchán, J. Andrés, J. Phys. Chem. B 2008, 112, 7153-7156.

[65] E. M. González, L. Guidoni, C. Molteni, Phys. Chem. Chem. Phys. 2009, 11, 4556-4563.

[66] S. Salzmann, M. R. Silva-Junior, W. Thiel, C. M. Marian, J. Phys. Chem. B 2009, 113, 15610-15618.

[67] M. G. Khrenova, T. Domratcheva, I. Schlichting, B. L. Grigorenko, A. V. Nemukhin, Photochem. Photobiol. 2011, 87, 564-573.

[68] Y.-W. Hsiao, J. P. Götze, W. Thiel, J. Phys. Chem. B 2012, 116, 8064-8073.

[69] N. C. Rockwell, L. Shang, S. S. Martin, J. C. Lagarias, Proc. Natl. Acad. Sci. U.S.A. 2009, 106, 6123-6127.

[70] M. Ikeuchi, T. Ishizuka, Photochem. Photobiol. Sci. 2008, 7, 1159-1167.

[71] N. C. Rockwell, S. S. Martin, K. Feoktistova, J. C. Lagarias, Proc. Natl. Acad. Sci. U.S.A. 2011, 108, 11854-11859.

[72] H. Li, A. D. Robertson, J. H. Jensen, Proteins 2005, 61, 704-721.

[73] D. C. Bas, D. M. Rogers, J. H. Jensen, Proteins 2008, 73, 765-783.

[74] J. W. Ponder, F. M. Richards, J. Comput. Chem. 1987, 8, 1016-1024. 
[75] R. Dennington, T. Keith, J. Millam, GaussView, Version 5, Semichem, Inc.: Shawnee Mission KS, 2009.

[76] H. Foerstendorf, C. Benda, W. Gärtner, M. Storf, H. Scheer, F. Siebert, Biochemistry 2001, 40, 14952-14959.

[77] B. Borucki, D. von Stetten, S. Seibeck, T. Lamparter, N. Michael, M. A. Mroginski, H. Otto, D. H. Murgida, M. P. Heyn, P. Hildebrandt, J. Biol. Chem. 2005, 280, 34358-34364.

[78] H. M. Strauss, J. Hughes, P. Schmieder, Biochemistry 2005, 44, 8244-8250.

[79] T. Rohmer, H. Strauss, J. Hughes, H. de Groot, W. Gärtner, P. Schmieder, J. Matysik, J. Phys. Chem. B 2006, 110, 20580-20585.

[80] W. D. Cornell, P. Cieplak, C. I. Bayly, I. R. Gould, K. M. Merz Jr., D. M. Ferguson, D. C. Spellmeyer, T. Fox, J. W. Caldwell, P. A. Kollman, J. Am. Chem. Soc. 1995, 117, 5179-5197.

[81] W. L. Jorgensen, J. Chandrasekhar, J. D. Madura, R. W. Impey, M. L. Klein, J. Chem. Phys. 1983, 79, 926-935.

[82] S. Dapprich, I. Komáromi, K. S. Byun, K. Morokuma, M. J. Frisch, J. Mol. Struct.: THEOCHEM 1999, 462, 1-21.

[83] M. J. Frisch, G. W. Trucks, H. B. Schlegel, G. E. Scuseria, M. A. Robb, J. R. Cheeseman, G. Scalmani, V. Barone, B. Mennucci, G. A. Petersson, H. Nakatsuji, M. Caricato, X. Li, H. P. Hratchian, A. F. Izmaylov, J. Bloino, G. Zheng, J. L. Sonnenberg, M. Hada, M. Ehara, K. Toyota, R. Fukuda, J. Hasegawa, M. Ishida, T. Nakajima, Y. Honda, O. Kitao, H. Nakai, T. Vreven, J. A. Montgomery, Jr., J. E. Peralta, F. Ogliaro, M. Bearpark, J. J. Heyd, E. Brothers, K. N. Kudin, V. N. Staroverov, R. Kobayashi, J. Normand, K. Raghavachari, A. Rendell, J. C. Burant, S. S. Iyengar, J. Tomasi, M. Cossi, N. Rega, J. M. Millam, M. Klene, J. E. Knox, J. B. Cross, V. Bakken, C. Adamo, J. Jaramillo, R. Gomperts, R. E. Stratmann, O. Yazyev, A. J. Austin, R. Cammi, C. Pomelli, J. W. Ochterski, R. L. Martin, K. Morokuma, V. G. Zakrzewski, G. A. Voth, P. Salvador, J. J. Dannenberg, S. Dapprich, A. D. Daniels, Ö. Farkas, J. B. Foresman, J. V. Ortiz, J. Cioslowski, D. J. Fox, Gaussian 09, Revision B.01, Gaussian, Inc.: Wallingford CT, 2009. [84] D. Bakowies, W. Thiel, J. Phys. Chem. 1996, 100, 10580-10594.

[85] R. Bauernschmitt, R. Ahlrichs, Chem. Phys. Lett. 1996, 256, 454-464. 
[86] M. E. Casida, C. Jamorski, K. C. Casida, D. R. Salahub, J. Chem. Phys. 1998, 108, 4439-4449.

[87] R. E. Stratmann, G. E. Scuseria, M. J. Frisch, J. Chem. Phys. 1998, 109, 8218-8224.

[88] M. A. L. Marques, E. K. U. Gross, Annu. Rev. Phys. Chem. 2004, 55, 427-455.

[89] A. Dreuw, M. Head-Gordon, Chem. Rev. 2005, 105, 4009-4037.

[90] M. E. Casida, J. Mol. Struct.: THEOCHEM 2009, 914, 3-18.

[91] S. Grimme, J. Comput. Chem. 2006, 27, 1787-1799.

[92] A. D. Boese, N. C. Handy, J. Chem. Phys. 2002, 116, 9559-9569.

[93] Y. Zhao, D. G. Truhlar, J. Phys. Chem. A 2006, 110, 13126-13130.

[94] H. Iikura, T. Tsuneda, T. Yanai, K. Hirao, J. Chem. Phys. 2001, 115, 3540-3544.

[95] T. Yanai, D. P. Tew, N. C. Handy, Chem. Phys. Lett. 2004, 393, 51-57.

[96] O. A. Vydrov, G. E. Scuseria, J. Chem. Phys. 2006, 125, 234109.

[97] O. A. Vydrov, J. Heyd, A. V. Krukau, G. E. Scuseria, J. Chem. Phys. 2006, 125, 074106.

[98] A. V. Marenich, C. J. Cramer, D. G. Truhlar, J. Phys. Chem. B 2009, 113, 6378-6396.

[99] M. R. A. Blomberg, P. E. M. Siegbahn, G. T. Babcock, J. Am. Chem. Soc. 1998, 120, 8812-8824.

[100] S. J. A. van Gisbergen, A. Rosa, G. Ricciardi, E. J. Baerends, J. Chem. Phys. 1999, 111, 2499-2506.

[101] D. Jacquemin, V. Wathelet, E. A. Perpète, C. Adamo, J. Chem. Theory Comput. 2009, 5, 2420-2435.

[102] D. Tozer, R. D. Amos, N. C. Handy, B. O. Roos, L. Serrano-Andrés, Mol. Phys. 1999, 97, 859-868.

[103] M. Head-Gordon, R. J. Rico, M. Oumi, T. J. Lee, Chem. Phys. Lett. 1994, 219, 21-29.

[104] J. B. Foresman, M. Head-Gordon, J. A. Pople, M. J. Frisch, J. Phys. Chem. 1992, 96, 135-149.

[105] H. Nakatsuji, Chem. Phys. Lett. 1978, 59, 362-364. 
[106] F. Neese, J. Chem. Phys. 2003, 119, 9428-9443.

[107] U. Ryde, Dalton Trans. 2007, 607-625.

[108] U. Ryde, L. Olsen, K. Nilsson, J. Comput. Chem. 2002, 23, 1058-1070.

[109] S. Yin, M. G. Dahlbom, P. J. Canfield, N. S. Hush, R. Kobayashi, J. R. Reimers, J. Phys. Chem. B 2007, 111, 9923-9930.

[110] Y. Mizutani, S. Tokutomi, K. Aoyagi, K. Horitsu, T. Kitagawa, Biochemistry 1991, 30, 10693-10700.

[111] Y. Mizutani, S. Tokutomi, T. Kitagawa, Biochemistry 1994, 33, 153-158.

[112] M. Gouterman, J. Chem. Phys. 1959, 30, 1139-1161.

[113] M. Wanko, M. Hoffmann, P. Strodel, A. Koslowski, W. Thiel, F. Neese, T. Frauenheim, M. Elstner, J. Phys. Chem. B 2005, 109, 3606-3615.

[114] R. Ahlrichs, M. Bär, M. Häser, H. Horn, C. Kölmel, Chem. Phys. Lett. 1989, 162, 165-169.

[115] TURBOMOLE V6.3 2011, a development of University of Karlsruhe and Forschungszentrum Karlsruhe GmbH, 1989-2007, TURBOMOLE GmbH, since 2007; available at: http://www.turbomole.com (accessed January 19, 2013).

[116] C. Hättig, J. Chem. Phys. 2003, 118, 7751-7761.

[117] B. W. Matthews, R. E. Fenna, M. C. Bolognesi, M. F. Schmid, J. Mol. Biol. 1979, 131, 259-285.

[118] C. Olbrich, J. Strümpfer, K. Schulten, U. Kleinekathöfer, J. Phys. Chem. Lett. 2011, 2, 1771-1776.

[119] S. Shim, P. Rebentrost, S. Valleau, A. Aspuru-Guzik, Biophys. J. 2012, 102, 649-660. 


\begin{tabular}{|c|c|c|c|}
\hline Functional & $\lambda_{\max }(\mathrm{Q})$ & $\lambda_{\max }($ Soret $)$ & $\mathrm{f}_{\mathrm{Q}} / \mathrm{f}_{\text {Soret }}$ \\
\hline BP86 & 1.91 & 3.15 & 1.48 \\
\hline BLYP & 1.90 & 3.13 & 1.48 \\
\hline B97D & 1.91 & 3.16 & 1.49 \\
\hline$\tau$-HCTH & 1.91 & 3.17 & 1.49 \\
\hline B3LYP & 2.05 & 3.20 & 1.65 \\
\hline PBE0 & 2.10 & 3.27 & 1.54 \\
\hline M06-HF & 2.26 & 3.77 & 1.04 \\
\hline LC-BLYP & 2.31 & 3.83 & 1.08 \\
\hline CAM-B3LYP & 2.20 & 3.54 & 1.19 \\
\hline LC- $\omega$ PBE & 2.29 & 3.78 & 1.08 \\
\hline CIS & 2.68 & 4.31 & 1.03 \\
\hline CIS(D) ${ }^{\lfloor b\rfloor}$ & 2.19 & 3.69 & - \\
\hline Exp. ${ }^{[\mathrm{c}]}$ & 1.77 & 3.26 & - \\
\hline \multicolumn{4}{|c|}{$\begin{array}{l}\text { [a] All QM/MM calculations based on the optimized BV Full+Site structure and carried out } \\
\text { with the default QM system (QM1) and, unless otherwise noted, the 6-31+G(d,p) basis } \\
\text { set. } \\
\text { [b] CIS(D) absorption maxima calculated with the 6-31G(d,p) basis set and obtained as } \\
\mathrm{S}_{0} \rightarrow \mathrm{S}_{1} \text { (Q band) and } \mathrm{S}_{0} \rightarrow \mathrm{S}_{2} \text { (Soret band) excitation energies. } \\
\text { [c] Experimental data from Ref. [20]. }\end{array}$} \\
\hline
\end{tabular}




\begin{tabular}{|c|c|c|c|c|c|c|}
\hline \multirow[t]{2}{*}{ Functional } & \multicolumn{2}{|c|}{$\lambda_{\max }(\mathrm{Q})$} & \multicolumn{2}{|c|}{$\lambda_{\max }($ Soret $)$} & \multicolumn{2}{|c|}{$\mathrm{f}_{\mathrm{Q}} / \mathrm{f}_{\text {Soret }}$} \\
\hline & QM1 & QM2 & QM1 & QM2 & QM1 & QM2 \\
\hline BP86 & 1.91 & 1.88 & 3.15 & 3.10 & 1.48 & 1.23 \\
\hline BLYP & 1.90 & 1.87 & 3.13 & 3.08 & 1.48 & 1.20 \\
\hline B97D & 1.91 & 1.88 & 3.16 & 3.11 & 1.49 & 1.21 \\
\hline$\tau-\mathrm{HCTH}$ & 1.91 & 1.89 & 3.17 & 3.12 & 1.49 & 1.22 \\
\hline B3LYP & 2.05 & 2.02 & 3.20 & 3.21 & 1.65 & 1.34 \\
\hline PBE0 & 2.10 & 2.06 & 3.27 & 3.26 & 1.54 & 1.24 \\
\hline M06-HF & 2.26 & 2.18 & 3.77 & 3.70 & 1.04 & 0.91 \\
\hline LC-BLYP & 2.31 & 2.24 & 3.83 & 3.78 & 1.08 & 0.96 \\
\hline CAM-B3LYP & 2.20 & 2.12 & 3.54 & 3.51 & 1.19 & 1.04 \\
\hline LC- $\omega$ PBE & 2.29 & 2.22 & 3.78 & 3.74 & 1.08 & 0.97 \\
\hline CIS & 2.68 & 2.64 & 4.31 & 4.26 & 1.03 & 0.98 \\
\hline
\end{tabular}




\begin{tabular}{|c|c|c|c|c|c|c|c|c|c|}
\hline \multirow[t]{2}{*}{ Functional } & \multicolumn{3}{|c|}{$\lambda_{\text {max }}(Q)$} & \multicolumn{3}{|c|}{$\lambda_{\max }($ Soret $)$} & \multicolumn{3}{|c|}{$\mathrm{f}_{\mathrm{Q}} / \mathrm{f}_{\text {Soret }}$} \\
\hline & $\mathrm{dzp}^{[\mathrm{b}]}$ & $\mathrm{dzdp}^{[\mathrm{c}]}$ & $\operatorname{tzp}^{[\mathrm{d}]}$ & $\mathrm{dzp}^{[\mathrm{b}]}$ & $\mathrm{dzdp}^{[\mathrm{c}]}$ & $\operatorname{tzp}^{[\mathrm{d}]}$ & $\mathrm{dzp}^{[\mathrm{b}]}$ & $\mathrm{dzdp}^{[\mathrm{c}]}$ & $\operatorname{tzp}^{[\mathrm{d}]}$ \\
\hline BP86 & 1.95 & 1.91 & 1.93 & 3.20 & 3.15 & 3.16 & 1.49 & 1.48 & 1.51 \\
\hline BLYP & 1.94 & 1.90 & 1.92 & 3.19 & 3.13 & 3.15 & 1.49 & 1.48 & 1.51 \\
\hline B97D & 1.95 & 1.91 & 1.93 & 3.21 & 3.16 & 3.18 & 1.49 & 1.49 & 1.52 \\
\hline$\tau-\mathrm{HCTH}$ & 1.95 & 1.91 & 1.93 & 3.22 & 3.17 & 3.18 & 1.49 & 1.49 & 1.53 \\
\hline B3LYP & 2.09 & 2.05 & 2.07 & 3.28 & 3.20 & 3.23 & 1.74 & 1.65 & 1.73 \\
\hline PBE0 & 2.13 & 2.10 & 2.11 & 3.34 & 3.27 & 3.29 & 1.65 & 1.54 & 1.59 \\
\hline M06-HF & 2.30 & 2.26 & 2.27 & 3.86 & 3.77 & 3.78 & 1.04 & 1.04 & 1.06 \\
\hline LC-BLYP & 2.35 & 2.31 & 2.32 & 3.91 & 3.83 & 3.86 & 1.07 & 1.08 & 1.09 \\
\hline $\begin{array}{l}\text { CAM- } \\
\text { B3LYP }\end{array}$ & 2.24 & 2.20 & 2.21 & 3.60 & 3.54 & 3.57 & 1.18 & 1.19 & 1.20 \\
\hline LC- $\omega$ PBE & 2.33 & 2.29 & 2.30 & 3.84 & 3.78 & 3.80 & 1.08 & 1.08 & 1.10 \\
\hline CIS & 2.72 & 2.68 & 2.70 & 4.41 & 4.31 & 4.36 & 1.01 & 1.03 & 1.02 \\
\hline \multicolumn{10}{|c|}{$\begin{array}{l}\text { [a] All QM/MM calculations based on } \mathrm{t} \\
\text { with the default QM system (QM1). } \\
\text { [b] dzp = The 6-31G(d,p) basis set. } \\
\text { [c] dzdp = The 6-31+G(d,p) basis set. } \\
\text { [d] tzp = The 6-311G(2df,p) basis set. }\end{array}$} \\
\hline
\end{tabular}


Table 4. Calculated absorption maxima $\left(\lambda_{\max }\right.$, in $\left.\mathrm{eV}\right)$ of the $\mathrm{Q}$ band of the BV chromophore in gas phase, SMD and protein environments. ${ }^{\text {[a] }}$

\begin{tabular}{|c|c|c|c|c|c|c|c|c|c|}
\hline & $\lambda_{\max }^{(1)}$ & $\lambda_{\max }^{(2)}$ & $\lambda_{\max }{ }^{(3)}$ & $\lambda_{\max }^{(4)}$ & $\lambda_{\max }^{(1)}$ & $\lambda_{\max }^{(2)}$ & $\lambda_{\max }^{(3)}$ & $\lambda_{\max }^{(1)}$ & $\lambda_{\max }{ }^{(2)}$ \\
\hline $\operatorname{Env}^{[\mathrm{b}]} \rightarrow$ & Gas & Gas & SMD & Protein & $\rightarrow$ & $\rightarrow$ & $\rightarrow$ & $\rightarrow$ & $\rightarrow$ \\
\hline $\mathrm{Geo}^{[\mathrm{c}]} \rightarrow$ & Gas & Protein & Protein & Protein & $\lambda_{\max }{ }^{(2)}$ & $\lambda_{\max }^{(3)}$ & $\lambda_{\max }^{(4)}$ & $\lambda_{\max }^{(4)}$ & $\lambda_{\max }{ }^{(4)}$ \\
\hline \multicolumn{10}{|l|}{ Functional } \\
\hline BP86 & 1.84 & 1.94 & 1.76 & 1.91 & 0.10 & -0.18 & 0.15 & 0.07 & -0.03 \\
\hline BLYP & 1.83 & 1.93 & 1.75 & 1.90 & 0.10 & -0.18 & 0.15 & 0.07 & -0.03 \\
\hline B97D & 1.84 & 1.95 & 1.76 & 1.91 & 0.11 & -0.19 & 0.15 & 0.07 & -0.04 \\
\hline$\tau$-HCTH & 1.85 & 1.95 & 1.76 & 1.91 & 0.10 & -0.19 & 0.15 & 0.06 & -0.04 \\
\hline B3LYP & 1.86 & 1.96 & 1.89 & 2.05 & 0.10 & -0.07 & 0.16 & 0.19 & 0.09 \\
\hline PBE0 & 1.91 & 2.00 & 1.93 & 2.10 & 0.09 & -0.07 & 0.17 & 0.19 & 0.10 \\
\hline M06-HF & 2.00 & 2.17 & 2.10 & 2.26 & 0.17 & -0.07 & 0.16 & 0.26 & 0.09 \\
\hline LC-BLYP & 2.03 & 2.20 & 2.12 & 2.31 & 0.17 & -0.08 & 0.19 & 0.28 & 0.11 \\
\hline CAM-B3LYP & 1.99 & 2.12 & 2.03 & 2.20 & 0.13 & -0.09 & 0.17 & 0.21 & 0.08 \\
\hline LC- $\omega \mathrm{PBE}$ & 2.03 & 2.18 & 2.11 & 2.29 & 0.15 & -0.07 & 0.18 & 0.26 & 0.11 \\
\hline $\mathrm{CIS}(\mathrm{D})^{\lfloor\mathrm{d}\rfloor}$ & 1.94 & 2.05 & - & 2.19 & 0.11 & - & - & 0.25 & 0.14 \\
\hline \multicolumn{10}{|c|}{$\begin{array}{l}\text { [a] Unless otherwise noted, all calculations carried out with the 6-31+G(d,p) basis set. } \\
\text { [b] Environment = Gas: QM calculations carried out in the gas phase. } \\
\text { Environment = SMD: QM calculations carried out using a SMD description of the protein. } \\
\text { Environment = Protein: QM/MM calculations carried out with the default QM system (QM1). } \\
\text { [c] Geometry = Gas: Calculations based on the B3LYP/6-31G(d,p) optimized BV structure. } \\
\text { Geometry = Protein: Calculations based on the QM/MM optimized BV Full+Site structure. } \\
\text { [d] CIS(D) absorption maxima calculated with the 6-31G(d,p) basis set and obtained as } \\
\mathrm{S}_{0} \rightarrow \mathrm{S}_{1} \text { excitation energies. }\end{array}$} \\
\hline
\end{tabular}




\section{Figure Captions}

Figure 1. Structure of the chromophore binding domain of Deinococcus radiodurans bacteriophytochrome in the Pr form.

Figure 2. Structure of the biliverdin IX $\alpha$ chromophore.

Figure 3. Atoms included in the default (QM1) and expanded (QM2) QM systems.

Figure 4. Calculated absorption maxima of the $\mathrm{Q}$ band of $\mathrm{DrBphP}$ for different protein structures in comparison with the experimental value (dashed line). ${ }^{[20]}$

Figure 5. Calculated absorption maxima of the Soret band of $\mathrm{DrBphP}$ for different

protein structures in comparison with the experimental value (dashed line). ${ }^{[20]}$ 


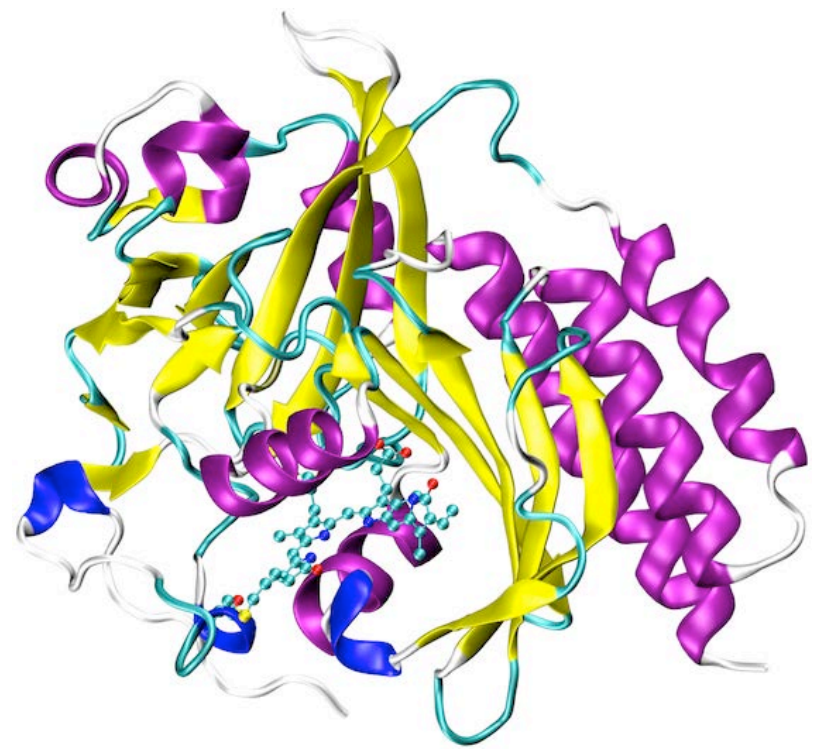

Figure 1

$36(40)$ 


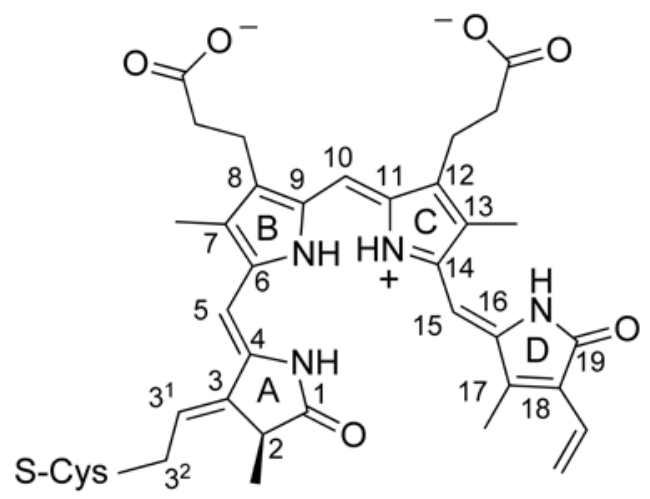

Figure 2 

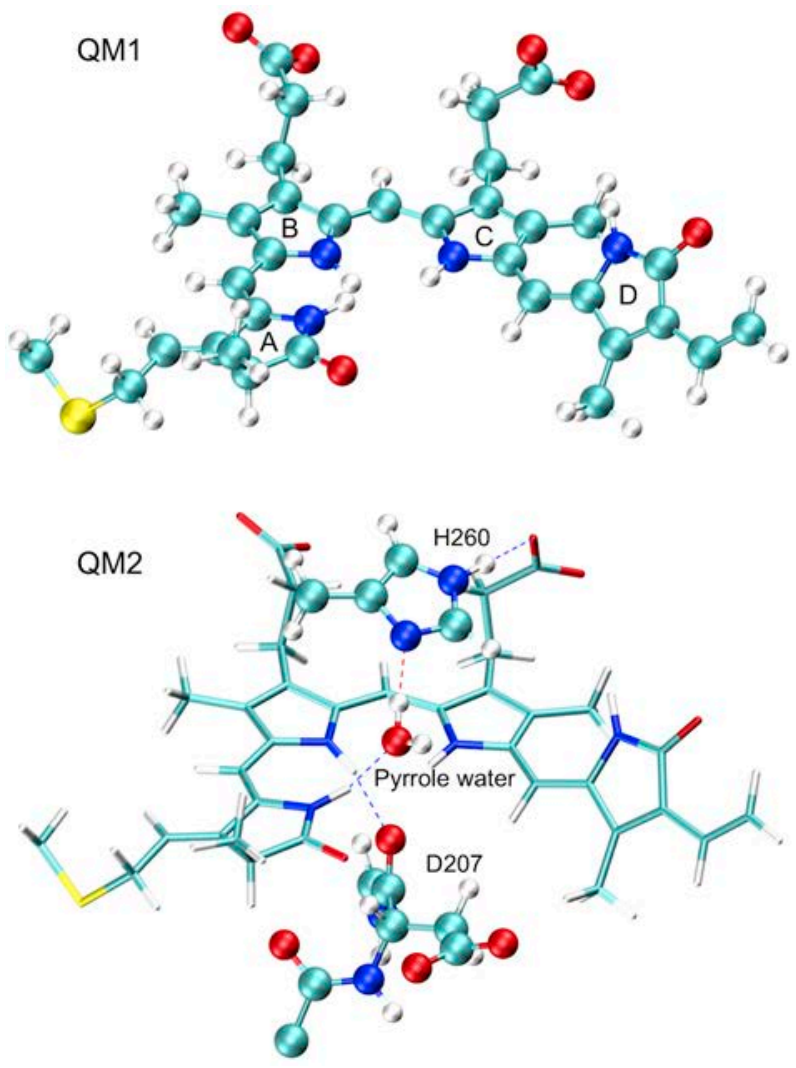

Figure 3 

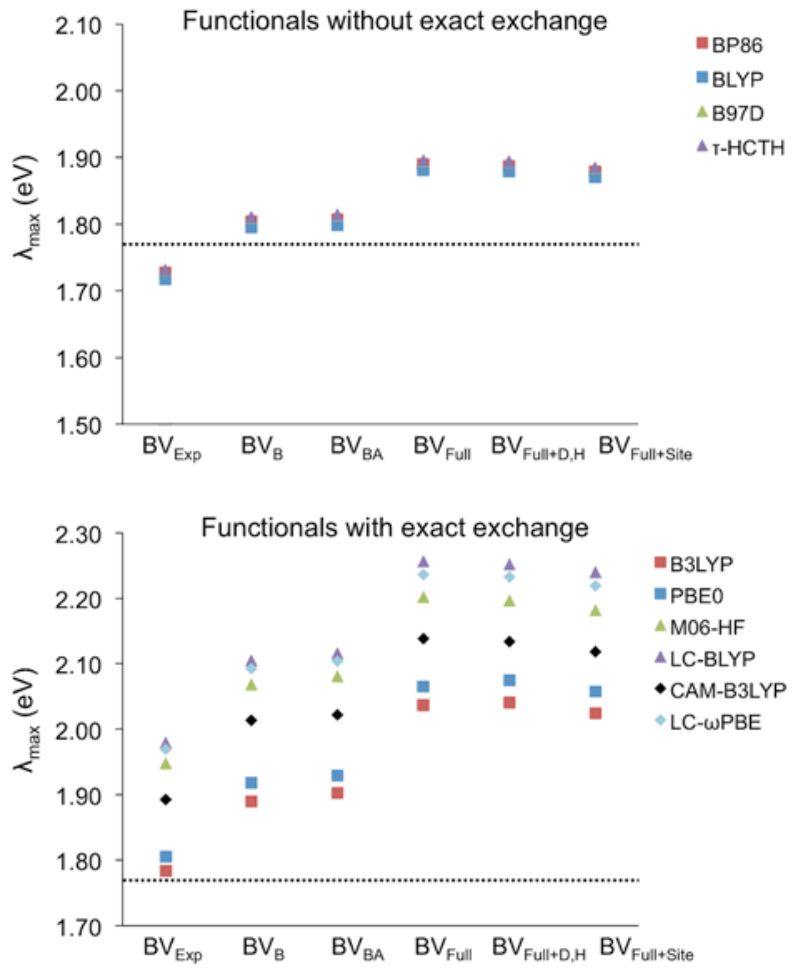

Figure 4 

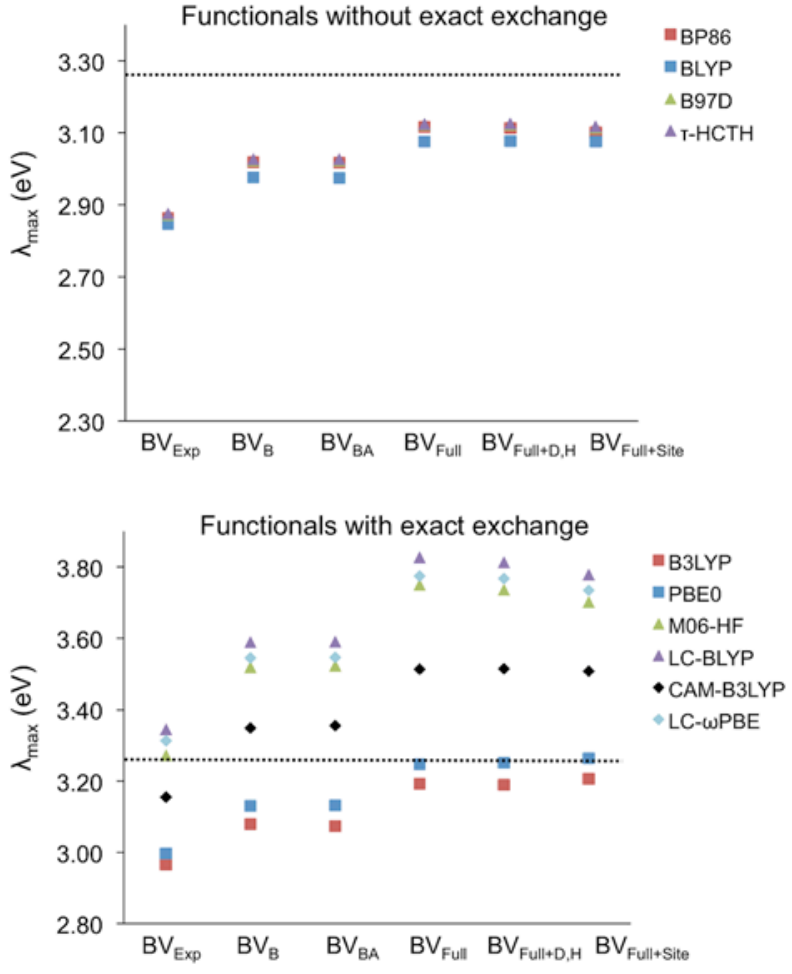

Figure 5 